\title{
EMC Measurement System Based on Software Defined Radio and Diagnostic Techniques
}

\author{
Rubén Tena Sánchez, Manuel Sierra Castañer \\ Centro de Investigación en Procesado de la Información \\ y Telecomunicaciones, ETSI Telecomunicación \\ Universidad Politécnica de Madrid \\ 28040, Madrid, Spain \\ rubents93@gr.ssr.upm.es, manuel.sierra@upm.es
}

\author{
L.J. Foged, D. Gray \\ MVG, Microwave Vision Group \\ lars.foged@mvg-world.com, Donald.Gray@mvg-us.com
}

\begin{abstract}
In a previous paper a new referenceless measurement set-up based on a reference antenna was used for characterizing the radiation of antennas in the planar scanner [1]. The method is based on using a low-cost receiver to retrieve the amplitude and phase of the signal. This paper explores the limitations of the method for different geometries and implements a multiprobe electromagnetic compatibility measurement system. Once the amplitude and phase are recovered, diagnostic techniques can be applied and also near-field to near or far-field transformations to calculate the field at distances defined by standards. The results demonstrate the good accuracy of the method in comparison with traditional electromagnetic compatibility laboratories.
\end{abstract}

\section{INTRODUCTION}

The growing of wireless communications is leading to the necessity of integration of the devices, thus the antenna is part of the overall design of the system. This presents plenty of advantages from the designing and manufacturing point of view. Nevertheless, the measurement of these integrated devices represent a real challenge, since the signal from the source is not accessible and therefore the phase can not be acquired.

Modern antennas or new wireless devices are not the only cases that represent a challenge when trying to get a phase reference for measurements. Electromagnetic compatibility (EMC) represents another example of devices for which it is not possible to access to a reference signal.

Classical measurement techniques either measure the signal in far-field or in near-field where amplitude and phase is necessary if a transformation is accomplished. Sometimes farfield measurements are not the solution, either the power received is very low or the sources can not be reconstructed if only the amplitude is acquired. On the contrary, nearfield measurements represent a full characterization of the radiated field by the AUT/DUT in amplitude and phase, but the conventional near field measurements can not face referenceless scenarios.

It is well known that there are phaseless techniques that deal with amplitude only methods to retrieve the phase of the field. Holographic techniques [2] may be a solution. It requires further hardware to generate the reference field, and due to overlapping in the spectral domain it may require a high sampling rate. Iterative methods between two scanning surfaces are also a possible solution, but the measurement time is increased and it is very sensitive to the initial guess and separation between surfaces (presence of local minimum). In [3] mean errors about $-35 \mathrm{~dB}$ where achieved by choosing the proper initial guess and separation between acquisition planes. Interferometry methods [4] or probe with multiple outputs [5] have also been tested in the literature. The drawback of interferometry is the necessity of extra hardware to process the in-quadrature and in-phase combination of signals and the risk of propagated errors. The errors may be minimized by using linear combination of signals at different positions, but the measurement time is increased.

In [1], a simple and cost-effective alternative method in comparison with the existing techniques was introduced. The method overcomes the complexity and cost of amplitude only methods and promising results were showed. The technique is based on the improvements of software defined radio (SDR) receivers architectures that allows over the air time domain measurements of the desired signals. The approach followed is different from amplitude only methods. Indeed, the SDR implements a phase recovery unit that exploits the radiation of the device to get a reference signal by using a reference antenna. This simplifies the process of retrieving the phase since Fast Fourier techniques can be applied to get the information. Nevertheless, there are some limitations mainly attributed to the interference of the reference antenna, although post-processing techniques can minimize this perturbation.

This paper aims to continue with the research done in the previous paper but considering different geometries to understand if some systems may present some advantages in order to reduce errors in the measurement. The second goal is to implement an EMC test case.

The paper is organized as follows: in Section II an explanation of the differences between the phase recovery applied to different geometries is explained. Section III will give some measurement results and will analyze the impact of the reference antenna for a spherical multiprobe system. Section IV will present a EMC test case. Finally, in Section V the conclusions and future lines are presented. 


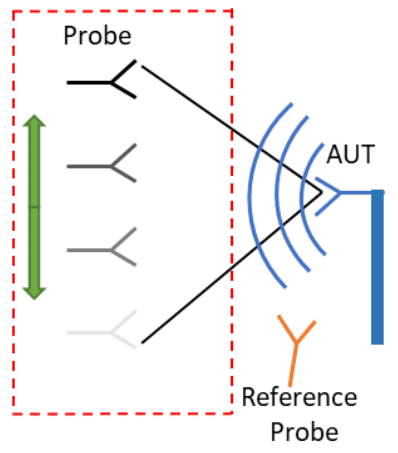

Fig. 1. Planar Scanning set-up

\section{DifFerent Geometries Comparison}

The main limitation regarding the location of the reference antenna is to keep its relative position with respect to the AUT fixed while scanning. Depending on which antenna is moved, AUT or probe, keeping the position of the reference antenna fixed with respect to the AUT may be challenging. Therefore, in this section different geometries are analyzed for the particular cases of our measurement systems to evaluate whether the set-up is feasible or not.

\section{A. Planar Scanning}

The AUT is fixed and the probe is moving in $\mathrm{x}, \mathrm{y}$-axis. There are some considerations regarding the reference antenna:

- It can not be placed behind the AUT because it would require a mounting structure to hold the reference that would depend on the AUT size. Moreover the power received would be very low.

- It could be placed between the AUT and the probe antenna but without blocking the line of sight between the probe and AUT.

- It is difficult to minimize the impact over the scanning probe while maximizing the SNR of the reference probe.

In order to fulfill these requirements, it has been tested experimentally that the optimum position would be beneath the AUT and pointing towards the AUT aiming to reject possible interferences coming from the probe and maximizing the power coming from the AUT, see Fig. 1. The errors are not only due to unstable reference channel but also due to contributions from the reference antenna that perturb the amplitude retrieved. Post-processing techniques like spacial filtering are effective in order to reduce the errors introduced by the reference antenna [1].

\section{B. Spherical Scanning}

One of the most widely used measurement systems due to its accuracy is the spherical near field acquisition. The particular spherical measurement system available in our anechoic chamber uses a roll over azimuth scanning system. Therefore the probe is fixed while the AUT is rotating over the different axis, see Fig. 3.

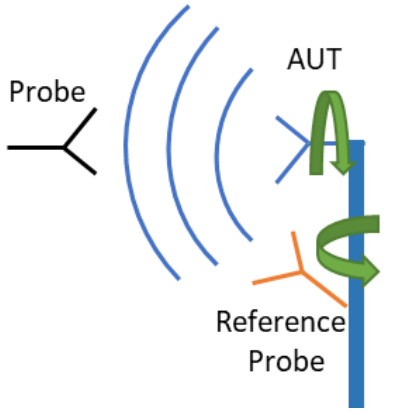

Fig. 2. Spherical Scanning set-up

The AUT rotation in roll over azimuth increases the complexity of the reference antenna method. Actually, the reference should be placed in a structure to rotate simultaneously with the AUT. Moreover there will be some directions for with the reference would interfere with the line of sight.

The last drawback makes the system very complex from the mechanical point of view, thus it was decided not to test the set-up for this system and evaluate other options.

\section{Multiprobe System}

The spherical multiprobe system presents some advantages over the other two geometries that can be exploited:

- There is not roll movement, therefore it is easier to implement than the spherical set-up.

- For a given cut, the system is stationary.

- Low impact of reference antenna for top probes.

- Some probes are disturbed by the reference in the line of sight. Nevertheless, if the reference is placed beneath de AUT, the probes affected by these interference will represent a small portion of the energy radiated by the device.

The intrinsic advantages of the multiprobe system make it suitable to test the proposed set-up. More specifically, the spherical multiprobe system "StarLab" from SATIMO will be used to test the accuracy of the method. Moreover, an EMC test case based on the multiprobe system will be tested, since the low-cost of the measurement system in combination with the receiver represents a good opportunity for measuring nonintentional radiations.

\section{Multiprobe Spherical SeT-UP}

The previous analysis showed that the spherical multiprobe system may present some advantages over other geometries. The first step before implementing the EMC test case is to validate the system.

The AUT used for validation was a standard gain horn antenna (SGH2000) from MVG. Without lost of generality the measurements were carried out at $2.3 \mathrm{GHz}$. Regarding the reference antenna, different options were evaluated, and eventually an electric sleeve dipole was used since it minimized the impact over the measurement sphere in terms of interference. The reference for the comparison will be the conventional measurement of the SGH2000 by the StarLab. 


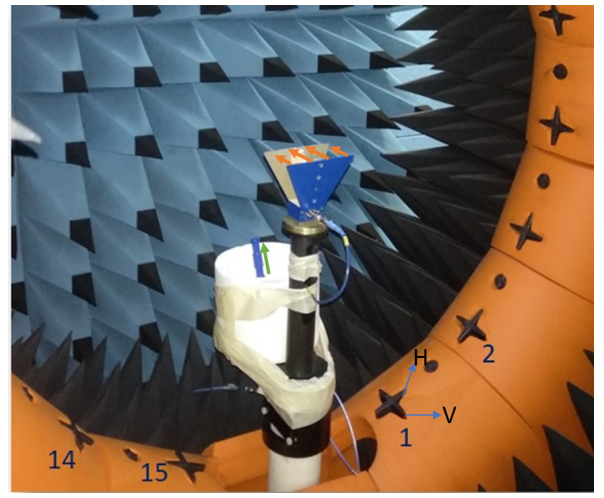

Fig. 3. Spherical Multiprobe Near Field set-up

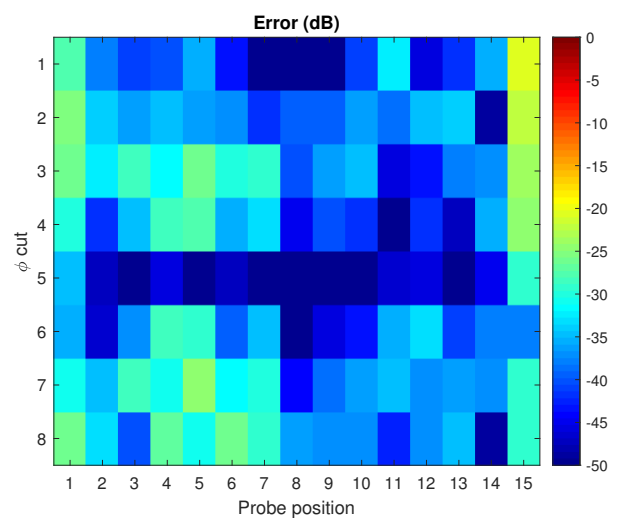

Fig. 4. Spherical Near Field error of V-component

Thereby, the comparison will represent the errors introduced by the reference antenna method in amplitude and phase.

Fig. 3 depicts the set-up used for measuring the radiation of the AUT. In the multliprobe system the AUT rotates in azimuth, therefore the interference may be critical for probes in the shadow region of the arch, when the reference antenna blocks the line of sight. Nevertheless these probes does not represent a significant part of the radiation of the device as it was stated in Section II.

The near-field error between the electric-field measured by the SDR and the StarLab measurement system has been computed by using Eq. (1). The electric-fields have been normalized to the maximum before the computation of the error.

$$
\operatorname{Error}(d B)=20 \log _{10}\left|E_{\text {StarLab }}-E_{S D R}\right|
$$

The step chosen in $\theta$ and $\phi$ was $22.5^{\circ}$. The last is traduced in 8 different azimuth positions and 15 different positions for $\theta$. Fig. 4 shows the errors for the different measurement points of the V-component. Each $\phi$ step corresponds to a clockwise rotation from the the first probe towards the fifteenth probe direction.

For this particular set of AUT/reference antenna it is clearly observed that the errors are mainly introduced for the probes

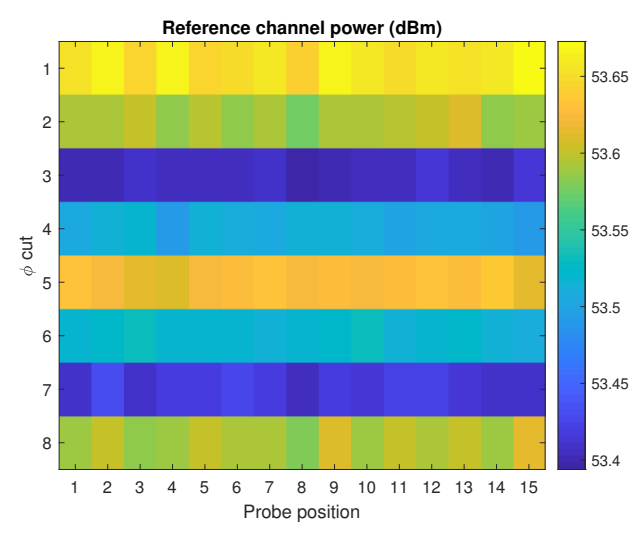

Fig. 5. Stability of reference channel

in the shadow region and also when the AUT is rotated in azimuth for the probes that are closer to the reference (from 1 to 6). A closer look to the error gives some insight of what is causing the pattern observed. The interference between probes and reference antenna will be larger for $\phi$ cuts where the V-component is not orthogonal from the radiation of the dipole. Moreover, the closer the dipole to the probe the larger the interference. The opposite is observed when the dipole radiation is orthogonal to the V-component of the field $\phi=90^{\circ}$ ( $\phi$ cut 5 in Fig. 4).

Another important figure of merit to analyze is the stability of the reference channel. This can be observed in Fig. 5. The power changes are minimal, which represents a very stable reference channel and this will be translated into low phase errors introduced in the system.

Even though there are some points of the measurement sphere that suffer from interference and thus errors, the mean error over the entire measurement surface is $-38 \mathrm{~dB}$ which represents a very low error since no post-processing is needed during the process compared to the planar case.

\section{EMC Test CAse in StarLab}

The validation of the proposed method in the spherical multiprobe system shows the potential of the technique if the main limitations are minimized. The low-cost equipment necessary to retrieve the amplitude and phase makes the system suitable for emission measurements. There are some results in the literature about near-field measurements for emission characterization, [6] is one example. Nevertheless they do not represent accurately the potential of the methods for source reconstruction and near-field to far-field transformation in order to calculate the radiated emissions at different distances.

The aim of this section is to validate the proposed method for EMC measurements, not only to calculate equivalent currents close to the device but also to calculate the radiated emissions at a given distance and compare the results with measurements done in a conventional EMC laboratory. In the particular case of this work the reference laboratory will be the High Technology and Homologation Center (CATECHOM) in the University of Alcala, Madrid. 


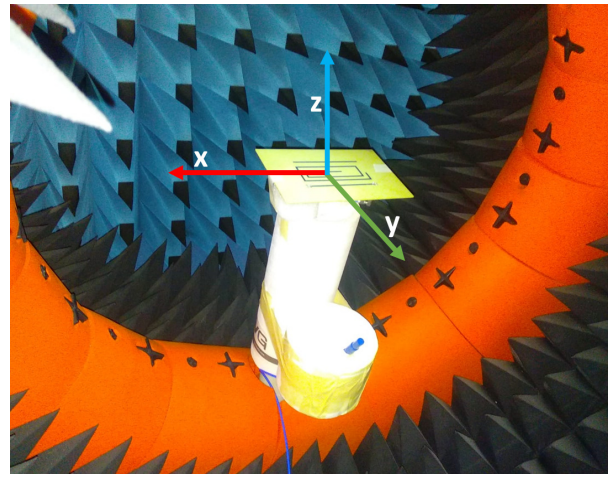

Fig. 6. EMC measurement set-up

To validate the system for EMC measurements, a reference DUT is necessary. For that purpose, the same sample as the one used in [6] will be characterized. The reference PCB size is $150 \mathrm{~mm}$ by $225 \mathrm{~mm}$ with substrate thickness of $2 \mathrm{~mm}$. One of the traces of the PCB is excited and the other ones are excited due to coupling. The PCB radiation efficiency is very low, indeed more than $99 \%$ of the power is absorbed by the load. Thus, it will represent a reliable test case for EMC characterization. The advantage of the PCB sample in contrast with other EMC devices is that conventional nearfield measurements can be performed in order to compare the results obtained and determine the accuracy of the proposed method.

\section{A. PCB Radiation Measurements}

The set-up used is based on the optimization performed in Section III, see Fig. 6. The PCB will be measured at the frequency of $2.3 \mathrm{GHz}$. It was intended to place the DUT exactly at the center of the measurement system, nevertheless there are some errors as will be observed in the source reconstruction of the PCB. Considering the frequency and size of the PCB the $\Delta \theta$ and $\Delta \phi$ steps are $7.5^{\circ}$. This means an oversampling factor of 3 in the StarLab measurement system.

The measured near-field radiation of both components by using the proposed technique in comparison with the conventional near-field measurements for $\phi=0^{\circ}$ are shown in Figs. 7 and 8 . The good agreement between both measurement systems can be seen in the low error represented in the figure according to Eq. (1). The mean error for both components, $\mathrm{H}$ and $\mathrm{V}$, over the whole measurement points is $-34.7 \mathrm{~dB}$ and $-35.5 \mathrm{~dB}$ respectively.

\section{B. Source Reconstruction}

The spherical wave expansion or the equivalent currents technique [7] may be applied to check whether it is possible to reduce the errors or not.

The first post-processing technique mentioned is a nearfield to near-field transformation by filtering spherical modes. According to the size of the DUT and Eq. (2), a total number of $15 \mathrm{~N}$-modes would be necessary to represent accurately the

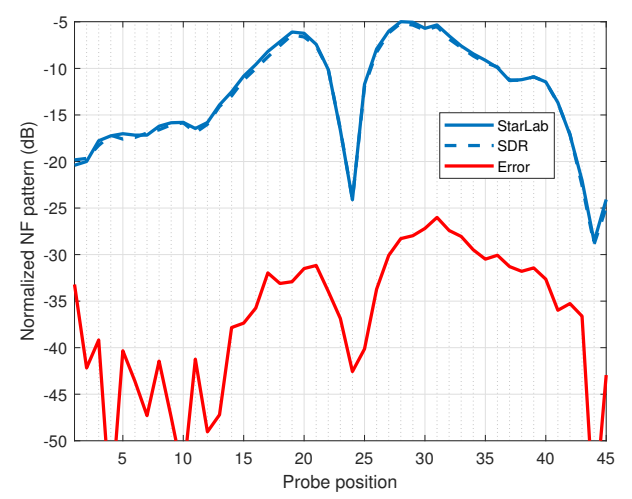

Fig. 7. Comparison of PCB near-field pattern for H-component

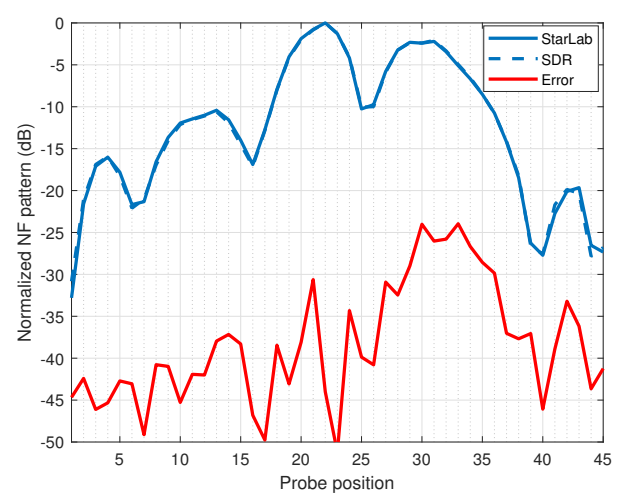

Fig. 8. Comparison of PCB near-field pattern for V-component

radiation from the device. Thus, the filtering was performed and the results compared with the reference electric-field.

$$
N=k r+10
$$

The second technique is based on the intrinsic filtering of equivalent source reconstruction. The software used to perform the source reconstruction was INSIGHT. The reconstruction surface was a box centered with the measurement coordinate system. The size was the same as the PCB but with an extra length of $\frac{\lambda}{5}$ in each dimension.

The mean error between the reference near-field measured in the StarLab system and the post-processed fields is shown in Table I.

In the case of equivalent sources an analysis of the error value shows that the error contributions are mapped into the box. The reason is that the errors are not very prominent and it is not possible to improve the results with the intrinsic filtering of equivalent currents. Modal filtering is not useful neither in this case because the small errors are mapped into coefficients that represent the radiation of the DUT itself. Thereby, this is the better result that can be achieved to reconstruct the sources and compute the radiated field at different distances.

The next step is to evaluate the source reconstruction done by INSIGHT. The comparison will evaluate the differences 
TABLE I

NF ERRORS

\begin{tabular}{lll}
\hline Electric field & H-component (dB) & V-component (dB) \\
\hline Raw SDR & -34.7 & -34.6 \\
\hline SWE filter & -34.98 & -34.5 \\
\hline Insight filter & -33.5 & -32.9
\end{tabular}

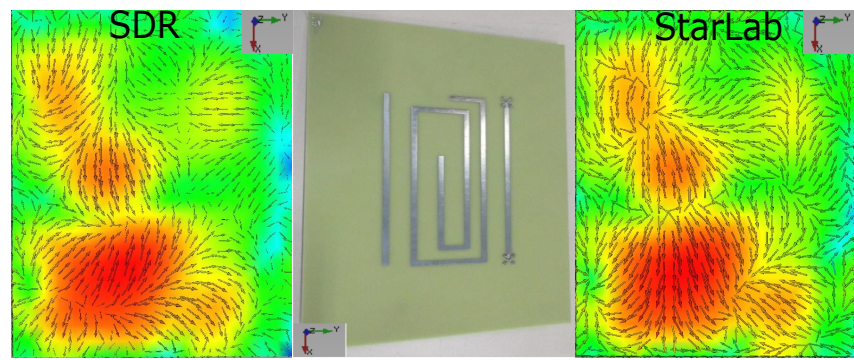

Fig. 9. Absolute value of reconstructed equivalent currents, Jeq

between the sources retrieved from the measurements done in the StarLab and the measurements performed with the referenceless system.

The triangular mesh of each reconstruction surface has been set to a resolution of $\frac{\lambda}{10}$ which represents the $8.6 \%$ and $5.7 \%$ of the minimum and maximum size of the PCB traces respectively. Fig. 9 depicts the absolute value of the reconstructed equivalent electric currents. There are some differences regarding the polarization of the currents. It was expected to observe that since the errors between near-fields is not negligible. Nevertheless, the agreement is very good in terms of intensity of the currents, which means that the measurement system is accurate enough in order to determine the location of the sources responsible of the emissions.

\section{Comparison with EMC Laboratory}

The reference PCB was measured in CATECHOM. The EMC procedure of the laboratory is based on sweeps in frequency with jumps of $60 \mathrm{kHz}$. An EMI peak detector with integration time of $50 \mathrm{~ms}$ per frequency is used. The measurement is performed in a cylindrical geometry, azimuth and z-axis sweep. Fig. 10 shows the set-up for the EMC characterization. It was intended to create an anechoic environment by covering the floor with absorbers, since the regular measurements performed in CATECHOM are done in a semianechoic environment.

The input power at port 1 of the PCB is $0 \mathrm{dBm}$. The measurements performed in CATECHOM will not be considered as the reference, since the laboratory uncertainty is very high, $\pm 5.2 \mathrm{~dB}$ with a confidence of $2 \sigma$. Instead, the StarLab measurements will be the reference for the comparison due to the reliability of the system.

The structures that may interfere with the measured radiation pattern will be simulated by importing the equivalent currents into CST and modelling the electromagnetic environment surrounding the PCB. In our particular case only the

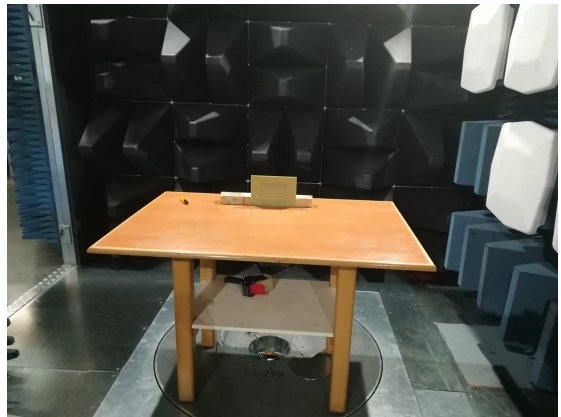

Fig. 10. CATECHOM Set-up

wooden structures will be included and any other effect will be considered negligible.

The first task to accomplish is to find the relative permittivity of the wooden parts. This value will depend on the specific conditions of the wood itself, but the permittivity will be considered the same for all the parts in the optimization process. The retrieval of the most likely permittivity of the material was done by trying to fit the near-field computation of the StarLab measurements at $3 \mathrm{~m}$ (including the wooden parts) with the CATECHOM measurements. Thereby, a sweep of the relative permittivity was done for theoretical values that depend on the conditions of the wood (1.2-6).

A final value of 1.6 was found to be optimum for the relative permittivity. Then, the SDR equivalent electric and magnetic currents calculated by INSIGHT were imported into CST as a near-field source and the wooden structures included in the simulation, see Fig. 11.

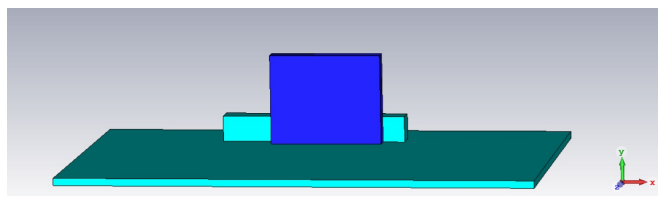

Fig. 11. CST model for full-wave simulation

Figs. 12 and 13 shows the results after including the effect of the wooden table and blocks on the radiation pattern at $3 \mathrm{~m}$. The Equivalent Error Signal (EES) has been computed by considering Eq. (3). It can be seen that the errors of the CATECHOM chamber with respect to the computed field from the StarLab measurements are within the uncertainty given by the laboratory. Moreover, the agreement between the StarLab computation and the results using the data from the referenceless set-up is very good. The mean error for both components is below $0.66 \mathrm{~dB}$ for $\theta \in\left[-90^{\circ}, 90^{\circ}\right]$.

$$
E E S(d B u V / m)=20 \log _{10}\left|\frac{|E|-\left|E_{r e f}\right|}{1 u V}\right|
$$

Thereby, it has been possible to optimize the reference antenna to minimize the impact over the measurement sphere and perform not only diagnostics as was shown in the source 


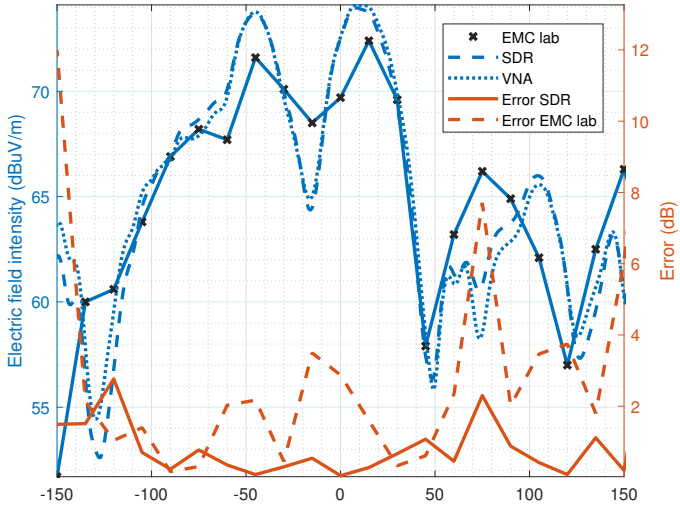

Fig. 12. Electric field intensity comparison at $3 \mathrm{~m} \mathrm{V-component}$

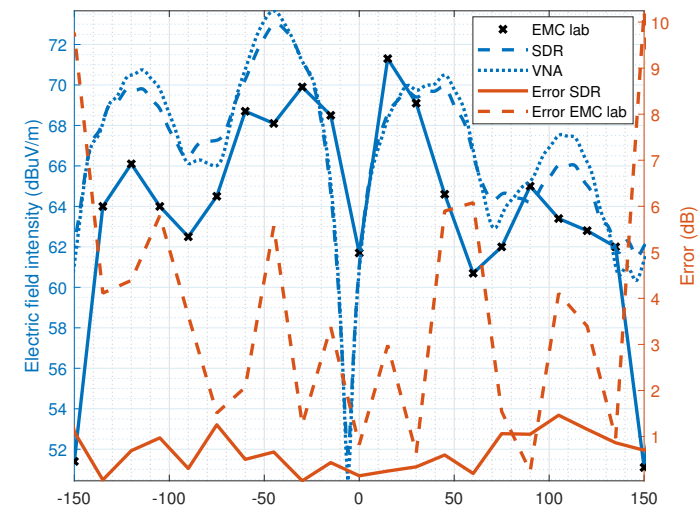

Fig. 13. Electric field intensity comparison at $3 \mathrm{~m} \mathrm{H}$-component

reconstruction but also the computation of the radiated emissions. The source reconstruction shows the good correlation between equivalent currents close to the PCB. The good agreement in the near-field propagation to $3 \mathrm{~m}$ demonstrate the potential of the system.

\section{CONCLUSions AND FUture Lines}

The development of a low-cost EMC multiprobe measurement system has been presented. First, different geometries has been compared to analyze the impact of the reference antenna in terms of amplitude and phase errors. Then the validation and optimization of the multiprobe set-up has been done by comparing the StarLab measurement of a SGH2000 with the electric field acquired by the referenceless system. Finally, a reference PCB has been used as a test case for EMC measurements. The near-field error of the referenceless acquisition in comparison with the StarLab system has been computed and it is low enough even without applying postprocessing techniques. Moreover the potential of the system has been shown by reconstructing the equivalent sources using INSIGHT and calculating the electric field at $3 \mathrm{~m}$ to compare it with a conventional EMC measurement performed in CATECHOM, Alcala de Henares. CST has been used as a computational electromagnetic tool to model the environment surrounding the PCB during the measurements performed in the EMC laboratory. The results obtained show the good agreement with respect to the reference and the potential of the system for radiated emissions measurements.

\section{ACKNOWLEDGEMENT}

Authors would like to acknowledge the Spanish Government, Ministry of Economy, National Program of Research, Development and Innovation for the support of this publication in the project FUTURE-RADIO "Radio systems and technologies for high capacity terrestrial and satellite communications in an hyperconnected world" (project number TEC201785529-C3-1-R).

\section{REFERENCES}

[1] R. Tena Sanchez and M. S. Castaner. "Evaluation of Software Defined Radio Receiver for Phaseless Near-Field Measurements". In: 2018 AMTA Proceedings, Williamsburg (2018), pp. 1-6.

[2] F. Las-heras, V. R. Antti, and E. Polit. "Reduced Set of Points in Phaseless Broadband Near-Field Antenna Measurement : Effects of Noise and Mechanical Errors". In: 10th European Conference on Antennas and Propagation (EuCAP), Davos (2016), pp. 1-5.

[3] J. F. Alvarez and O. Breinbjerg. "Towards Planar Phaseless Near-Field Measurements of ESA' s JUICE Mission $600 \mathrm{GHz}$ SWI Reflector Antenna”. In: AMTA 2016 Proceedings, Austin, TX, USA (2016), pp. 1-6.

[4] A. Paulus, J. Knapp, and T. F. Eibert. "Utilizing partial knowledge of phase differences in convex optimization for amplitude-only near-field far-field transformation". In: 2017 11th European Conference on Antennas and Propagation, EUCAP 2017 (2017), pp. 3766-3770.

[5] M. Spang, T. Stoeckel, G. Schubert, M. Albach, and E. Fields. "Application of Probes with Multiple Outputs on Probe-Compensated EMC Near-Field Measurements". In: 2010 IEEE International Conference on Industrial Technology, Vina del Mar (2010), pp. 188-193.

[6] P. Anders, A. P. Mynster, and M. Sørensen. "Validation of EMC near-field scanning amplitude and phase measurement data". In: International Symposium on Electromagnetic Compatibility - EMC EUROPE, Rome (2012), pp. $1-6$.

[7] L. J. Foged, L. Scialacqua, F. Mioc, F. Saccardi, P. O. Iversen, L. Shmidov, R. Braun, J. L. A. Quijano, and G. Vecchi. "Echo Suppression by Spatial-Filtering Techniques in Advanced Planar and Spherical Near-Field Antenna Measurements". In: IEEE Antennas and Propagation Magazine 55 (2013), pp. 235-242. 